\title{
Biological Investigation Using Scanning Probe Recognition Microscopy
}

\author{
Qian Chen, Virginia Ayres, Lalita Udpa \\ Dept. of Electrical and Computer Engineering \\ Michigan State University \\ East Lansing, MI 48824
}

\begin{abstract}
Direct investigation of, and interaction with, biological objects at the macromolecular level will provide insight into multiple physical regulatory processes. Scanning probe microscopy (SPM) techniques have the potential to provide a direct interaction with living specimens at the macromolecular scale. In the present research we will discuss the site-specific recognition techniques that are appropriate for biological objects of different shapes in the SPM images. Moment-based shape features which are scale and translation invariant are successfully used to distinguish tubular objects from globular objects. Among the globular objects, white blood cells can be clearly classified from red blood cells by applying twodimensional continuous wavelet transform (CWT), which can represent information of objects in multiple scales. The scan plan for site-specific investigation can then be determined by employing multi-scale edge detection techniques on the result.
\end{abstract}

Keywords-moment-based shape feature; continuous wavelet transform; site-specific investigation

\section{INTRODUCTION}

Scanning Probe Microscopy (SPM) techniques have found widespread use in recent years largely due to its ability to provide high resolution images of specimens. But the manipulation of scanning probe microscopy tip in the $x-y$ plane is still a significant challenge, since a realistic solution requires techniques in image processing, pattern recognition and control. This problem has attracted attention of many researchers because of its importance in both nanotechnology and biomedical engineering.

Moment based approaches have been widely used for shape recognition in many applications [5]. In this paper, normalized central moments are successfully used to distinguish biological objects of two different shapes, namely tubular and globular. Further, this is combined with a method based on 2-D continuous wavelet transform to classify leukocytes (white blood cells) from erythrocytes (red blood cells) [1]. A brief introduction of this method along with some improvements to the decision rule and experimental analysis are also included in this paper.

\section{METHODS}

The crucial problem in the discrimination of biological objects is to determine the main dissimilarities between different objects. The main difference between tubular objects and globular objects is the shape; we use moment-based shape features to classify them. In the next step we distinguish leukocytes from erythrocytes, which both have globular shapes with the main difference that leukocytes have a nucleus and erythrocytes have no nucleus. The two-dimensional continuous wavelet transform is used to detect this difference. Figure. 1 shows the images of some typical biological objects discussed in this paper.

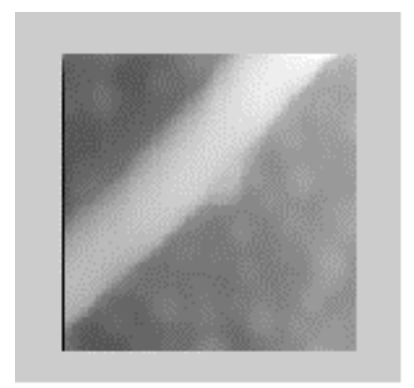

(a)

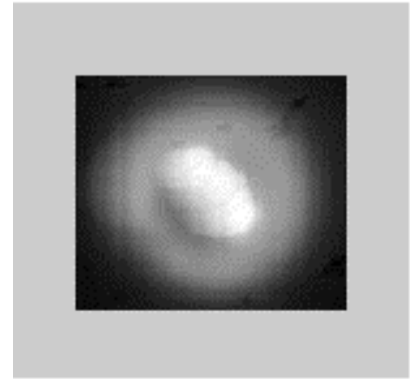

(c)

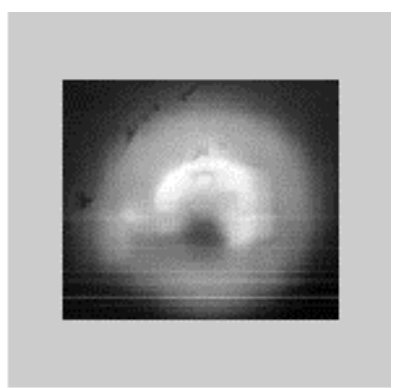

(b)

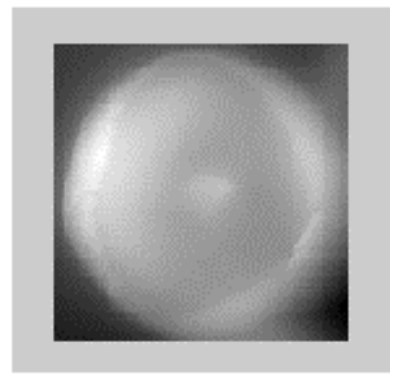

(d)
Figure 1. Typical biological objects (a) tube; (b) neutrophil leukocyte; (c) monocyte leukocyte; (d) erythrocyte

\section{A. Moment-based shape feature}

For 2-D discrete function $f(x, y)$, the moment of order $(p+q)$ is defined as [3]

$$
m_{p q}=\sum_{x} \sum_{y} x^{p} y^{q} f(x, y)
$$

The central moments can be expressed as

$$
\mu_{p q}=\sum_{x} \sum_{y}(x-\bar{x})^{p}(y-\bar{y})^{q} f(x, y)
$$

Where 


$$
\bar{x}=\frac{m_{10}}{m_{00}}, \quad \bar{y}=\frac{m_{01}}{m_{00}}
$$

The normalized central moments are defined as

$$
\eta_{p q}=\frac{\mu_{p q}}{\mu_{00}^{\gamma}}
$$

Where

$$
\gamma=\frac{p+q}{2}+1
$$

Since these moments are inherently integral-based features, using moment-based shape feature can reduce the effect of uncorrelated noise. Besides, moments are very easy to calculate, no prior information is needed except for segmented images of the objects. It is proven that normalized central moments are translation and scale invariant, which means that when we use the normalized central moments as shape feature, this feature remain unchanged if that shape undergoes any combination of change of size and change of position[2]. These properties make normalized central moments become a good choice to classify the tubular objects from globular objects.

\section{B. Continuous wavelet transform}

The 2-D continuous wavelet transform of function $f(x, y)$ is given by

$$
w\left(\sigma, \tau_{1}, \tau_{2}\right)=\frac{1}{\sigma} \iint f(x, y) \psi^{*}\left(\frac{x-\tau_{1}}{\sigma}, \frac{y-\tau_{2}}{\sigma}\right) d x d y
$$

Where $\sigma$ is the scale parameter, $\tau_{1}, \tau_{2}$ are the translation parameters, $*$ denotes the complex conjugation, and $\psi$ is the generating wavelet function.

The main use of 2-D continuous wavelet transform is to detect and analyze features of images of multiple scales. The scale parameter $\sigma$ plays an important role in the transform. At each scale, wavelet transform computes the similarity of the image data with the wavelet kernel at the selected scale $\sigma$. Thus, by choosing the optimal value of $\sigma$, we can get the relevant details from the image that are necessary to perform a particular recognition task across multiple scales.

This paper uses the differential Gaussian mother wavelet function for the 2-D continuous wavelet transform, whereby the edge information of images is enhanced. By drawing a radial line through the center of mass of the images, it is clear that leukocytes have 4 or more edges and erythrocytes have no more than 2 as shown in Figure 2. We count the number of zero-crossing in Figure 2 to represent the information of edges. The final decision is based on the following decision rule.

$$
\begin{array}{ll}
\diamond & \text { If } \mathrm{n}>=6, \text { object is a leukocyte } \\
\diamond & \text { If } \mathrm{n}<=4 \text {, object is an erythrocyte } \\
\diamond & \text { If } 4<\mathrm{n}<6, \text { object is unknown }
\end{array}
$$

Two orthogonal radial lines are selected for analyses so that the decision rule is more robust. For instance, if one of the decisions is unknown cell, the final decision is chosen based on the zero crossings of the other line. If one decision is leukocyte and the other decision is erythrocyte, the final decision is 'unknown'.

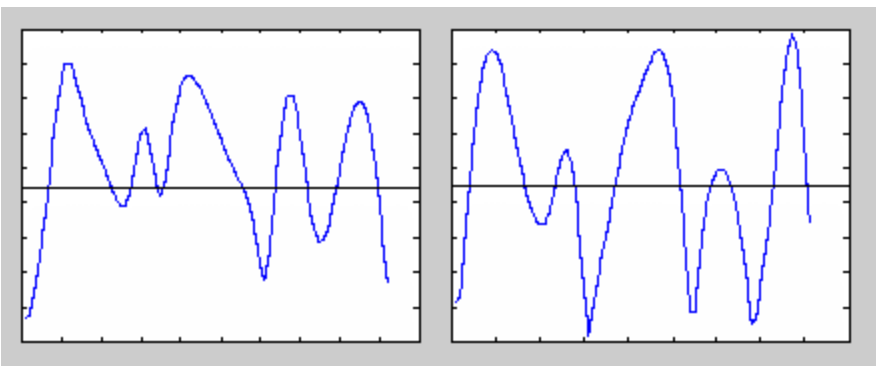

(a)

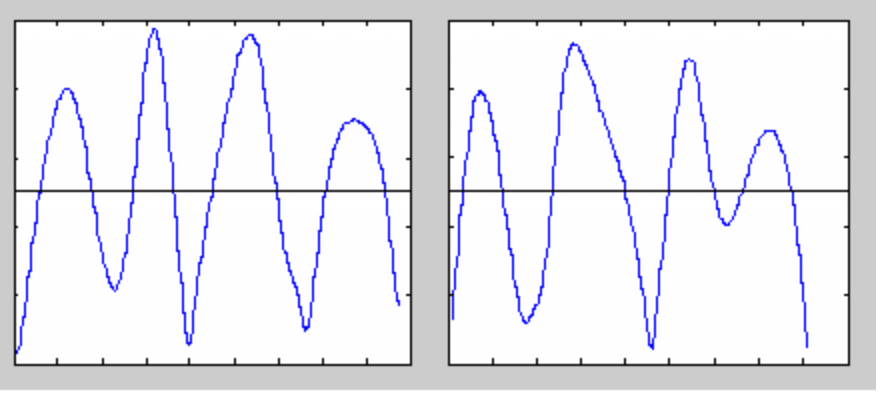

(b)

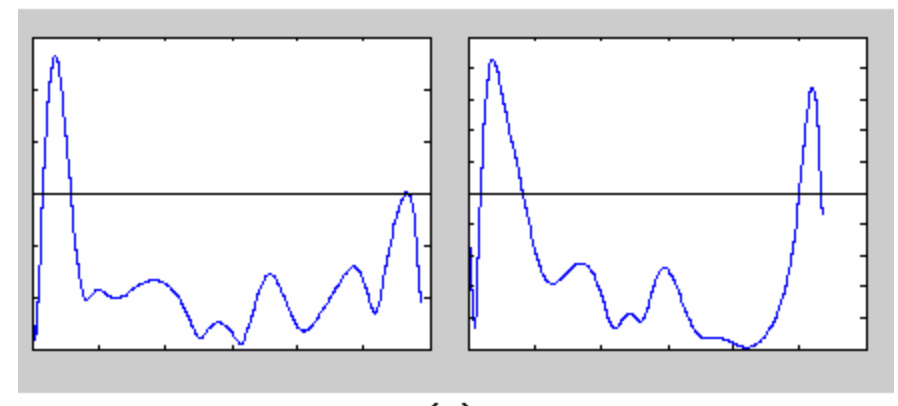

(c)

Figure 2. Grey level of horizontal and vertical lines of 2-D continuous wavelet transformed images (a) neutrophil leukocyte; (b) monocyte leukocyte; (c) erythrocyte

\section{EXPERIMENTAL RESULTS}

Scanning probe microscopy is used to generate Atomic Force Microscope (AFM) images of some biological specimens. 30 sample images were chosen and segmented. These images are used to test the accuracy of methods discussed above. There were 18 tubular objects and 12 global objects in these images.

\section{A. Tubular objects vs. globular ojbects}

By comparing the normalized central moments of different orders, we found moments of order $\mathrm{p}=2, \mathrm{q}=2$ were optimal to classify tubular objects from globular objects, as shown in Figure 3. The normalized central moments of other orders are either too complex to calculate or show poor performance. 


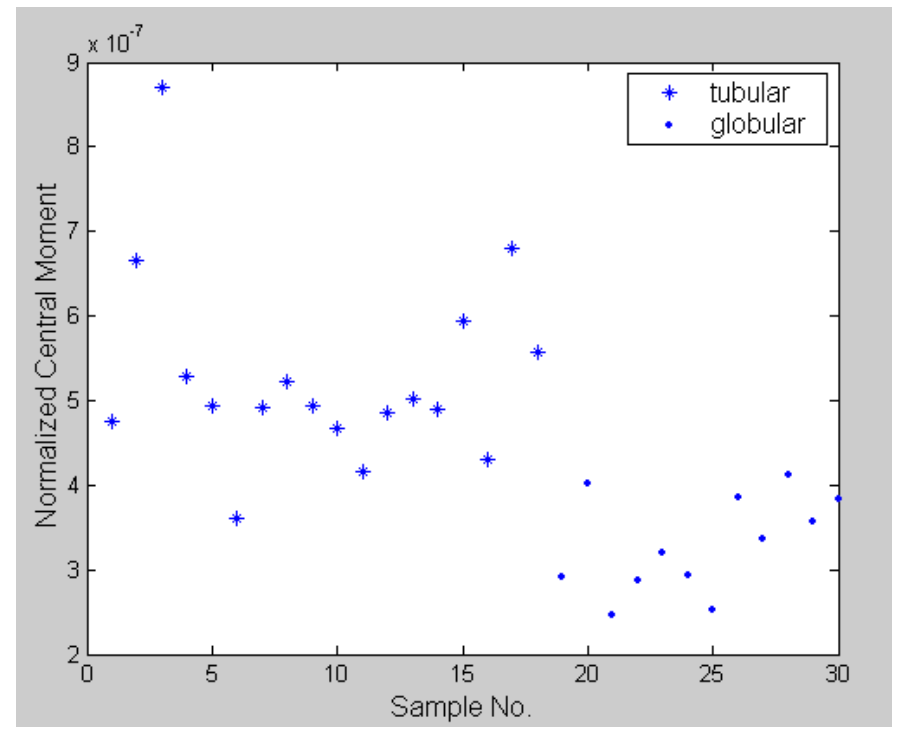

Figure 3. Comparison of nomalized central moment under order $(p=2, q=2)$

When threshold is chosen to be 4E-7, the result of this moment-based classifier is shown in table 1 .

TABLE 1. CONFUSION MATRIX OF MOMENT-BASED CLASSIFIER

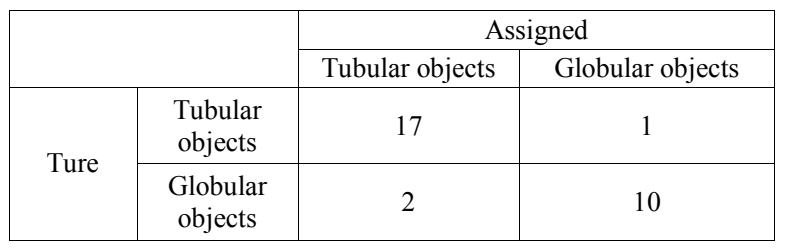

Table. 1 shows that 3 out of 30 objects are misclassified. By analyzing misclassified objects, we find that when segmented tubes are relatively short and occupy most areas of these images, their normalized central moments are close to that of globular objects. It will cause classification error. This kind of error can be avoided by getting more background areas during tube segmentation.

\section{B. leukocytes vs. erythrocytes}

The 12 globular objects include 6 leukocytes and 6 erythrocytes. After choosing the scale parameter appropriate to the real size of each object, we can get the 2-D continuous wavelet transform of these images. Using the decision rule discussed above, all the erythrocytes can be correctly assigned, and only one of the 6 leukocytes is misclassified.

The only misclassified leukocyte is once again due to error in segmentation of two closely attached cells. Thus, this method is suitable for images that are fully detached.

\section{DISCUSSION}

Methods have been proposed to classify some typical biological objects and the results on experimental AFM images have been reported and discussed. These objects with obvious differences can be classified easily with high accuracy. Work is currently underway to extend these methods to distinguish those objects with subtle dissimilarities, such as the monotype leukocytes and the neutrophil leukocytes. A simple extension is to combine two or more features to improve the accuracy of classification.

It is proven that normalized central moments are scale invariant, and 2-D continuous wavelet transform allows multiscale analysis of images. Thus, these two methods can be applied to analyze biological objects of any scale. But at present, the scale parameter of the continuous wavelet transform still needs to be decided by human observation and experiments. Work related to automatic determination of object scale is also in progress.

Moment-based shape features have the property of robustness under noise and high accuracy, but it needs a long processing time because of the complex computation. New schemes including both hardware and software are used to make the computation more efficient [4] since the speed requirement is critical for online implementation.

\section{REFERENCES}

[1] B. Goolsby, Q. Chen, L. Udpa, Y. Fan, R. Samona, B. Bhooravan, F. M. Salam, D. H. Wang, and V. M. Ayres, "Scanning Probe Microscopy with Landmark Referenced Control For Direct Biological Investigations," accepted for publication, J. Nanosci. Nanotech. (2003).

[2] R.J. Prokop and A.P. Reeves, "A Survey of Moment-Based Techniques for Unoccluded Object Representation and Recognition," CVGIP Graphical Models and Image Processing, vol. 54, no. 5, pp. 438 - 460, 1992.

[3] Rafael C. Gonzalez and Richard E. Woods, "Digital Image Processing," Addison-Wesley, 1992.

[4] Judit Martínez and Federico Thomas, "Efficient Computation of Local Geometric Moments," IEEE Transactions on Image Processing, Vol. 11, no. 9, pp. 1102-1111, September, 2002.

[5] P Reuze, JL Coatrieux, LM Luo, JL Dillenserger, " $A$ 3-d moment based approach for blood vessel detection and quantification in MRA," Technology and Health Care, 1, pp. 181-188, 1993. 